“C 2002 IEEE. Personal use of this material is permitted. Permission from IEEE must be obtained for all other uses, in any current or future media, including reprinting/republishing this material for advertising or promotional purposes, creating new collective works, for resale or redistribution to servers or lists, or reuse of any copyrighted component of this work in other works." 


\title{
An insight into the interplay between culture, conflict and distance in globally distributed requirements negotiations
}

\author{
Daniela E. Damian* and Didar Zowghi† \\ *University of Victoria, BC, Canada \\ danielad@cs.uvic.ca \\ $\dagger$ University of Technology, Sydney, Australia \\ didar@it.uts.edu.au
}

\begin{abstract}
There is an increasing interest in research addressing issues of global software development. Specifying software requirements is a communication-intensive collaborative activity that is increasingly performed across cultural, language and time zone boundaries. While inadequate communication significantly impacts the bridging of geographical distance between stakeholders, the cultural differences cannot be considered less significant. Findings from two global software development organizations enables us to present a model of impact of distance and the affected requirements activities due to problems of cultural diversity, inadequate communication, knowledge management and time differences. This evidence provides an important insight into the interplay between culture and conflict as well as the impact of distance on the ability to reconcile different viewpoints with regards to requirements and requirements processes.
\end{abstract}

\section{Requirements negotiations in global software development}

Software engineering is witnessing a transition from the traditional co-located form of development to a form in which global software teams collaborate across national borders. Research increasingly reports about projects developed between USA and India [11] as well as other continents such as Asia and Europe [3]. Findings emphasize major problems in communication and coordination, activities that are critical during early phases of strategic planning, requirements gathering, analysis and negotiation.

Requirements Engineering (RE) research in particular has yet to address the challenges faced by multi-site organizations. Previous reports only describe global projects where the requirements gathering and analysis were performed during face-to-face meetings at the client site, and the information communicated to the development staff [16]. It is equally important to understand and describe the impact of distance in projects where cross-functional stakeholder teams define the software requirements in global structures. Global organizations face challenges in enabling effective ongoing communication between headquarters and remote development sites. Although weekly meetings are needed for strategic planning and negotiations of tradeoffs between competing demands, organizations often don't have the luxury of arranging face-to-face requirements meetings on an ongoing basis. As a result, distance may exacerbate fundamental $\mathrm{RE}$ problems such as poor communication among stakeholders, as well as those due to factors of political, organizational and social nature.

While recent research investigated distributed requirements negotiations in a controlled setting [6], more evidence about multi-site organizations and $\mathrm{RE}$ at a distance needs to be gathered in the software industry. Given the relative novelty of the phenomenon and the paucity of research done in this area, it is important to conduct field investigations to understand the impact of problems of communication and coordination on RE activities, to identify challenges faced by multi-site organizations, and to formulate recommendations to overcome problems associated with distance. Practitioners need to be aware of such findings in order to cope with distance, when co-located development is not an option.

In this paper we report the findings of a case study of two multi-site development organizations where groups of customers, product management and engineering specified requirements from remote locations. We describe the challenges faced by the stakeholders in activities such as requirements elicitation, analysis, negotiation and specification. The specific focus of the paper is on an aspect that is gaining increased interest in the literature on global collaboration, i.e. the interplay between conflict and culture in the distributed teams.

The paper begins by a description of the research question and methodology in Section 2, followed by background information on the two multi-site organizations in the case study. In Section 4, requirements engineering challenges due to distance are described in the form of a three-layer model. It identifies four known problems of global software development, the specific challenges identified in this study, as well as the requirements engineering activities directly affected by these challenges. A common theme in the evidence from 
both multi-site organizations is the interplay between conflict, culture and distance in global requirements negotiations, aspect discussed in detail in Section 5.

\section{Research Methodology}

Our research question was "what is the impact of stakeholders' geographical distribution on RE activities in global software development?" The goal was to understand how requirements were developed in multi-site development organizations; to understand the challenges faced, strategies and technologies used to overcome these challenges.

The research method was the case study as described in [22]. The unit of analysis was the multi-site software development organization. The model of impact as well as the evidence about culture and conflict discussed in this paper is grounded in data collected at two multi-site organizations with headquarters in US and development sites in Australia. The organizations names are fictitious to preserve confidentiality.

Grounded theory methods [20] (comparative analysis within each organization and between the two organizations) were used to analyze the data. We spent over seven months in the first organization ("Global Development Software”, GDS henceforth), and findings from the first organization were described in [7]. This was followed by a period of five weeks of focused interviews at the second organization ("Software Production International", SPI henceforth). Continued collaboration with GDS allowed us to return to GDS and compare "backwards" and strengthen our findings to reflect aspects from both organization. One common theme that emerged was the interplay between conflict and culture in the distributed teams.

\section{Background information on the two organisations}

The two organizations were chosen since they provided a similar still different geographical distribution of stakeholders, and thus their comparison could serve to ground theoretical statements about the impact of stakeholders' geographical distribution on defining requirements in multi-site organizations.
The two multi-site software organizations were similar in that the requirements activities involved frequent and significant communication of two major stakeholder groups located on two different continents, Australia and USA. They were different in that at GDS, unlike SPI, there was a long-standing working relationship between the two stakeholder groups, as well as significantly more domain knowledge in product development, shared by the interested parties.

GDS has the development located in a major laboratory in Sydney and several other cities in Australia. The Business Management (BM) is located in USA, and customer segments world-wide. Major decisions are taken in weekly meetings involving three to five development management staff in Australia and four US-based BM members.

Similarly, at SPI development is located in two major Australian cities and a team of five belong to the Project Office team in one US location, while customers are located in five US locations. These stakeholder groups had not worked together on previous projects.

Both organizations have used a mix of communication technologies to support their collaboration over distance, including email, teleconferencing, videoconferencing and NetMeeting.

\section{RE challenges due to distance}

Our findings are described in the form of a model of RE challenges due to geographical distribution of stakeholders, illustrated in Figure 1. In the model, the top layer describes what we identified to be four major problems of geographical distribution of stakeholders. They align with findings of previous research of global software development [11]:

- Inadequate communication. Distance introduces barriers to informal and face-to-face communication, and the stakeholders' communication is dependent on the quality of using synchronous or asynchronous electronic communication tools. In this study, interest groups (customers, business management, and developers) did not communicate effectively and each sought to exert power and influence over the others. 


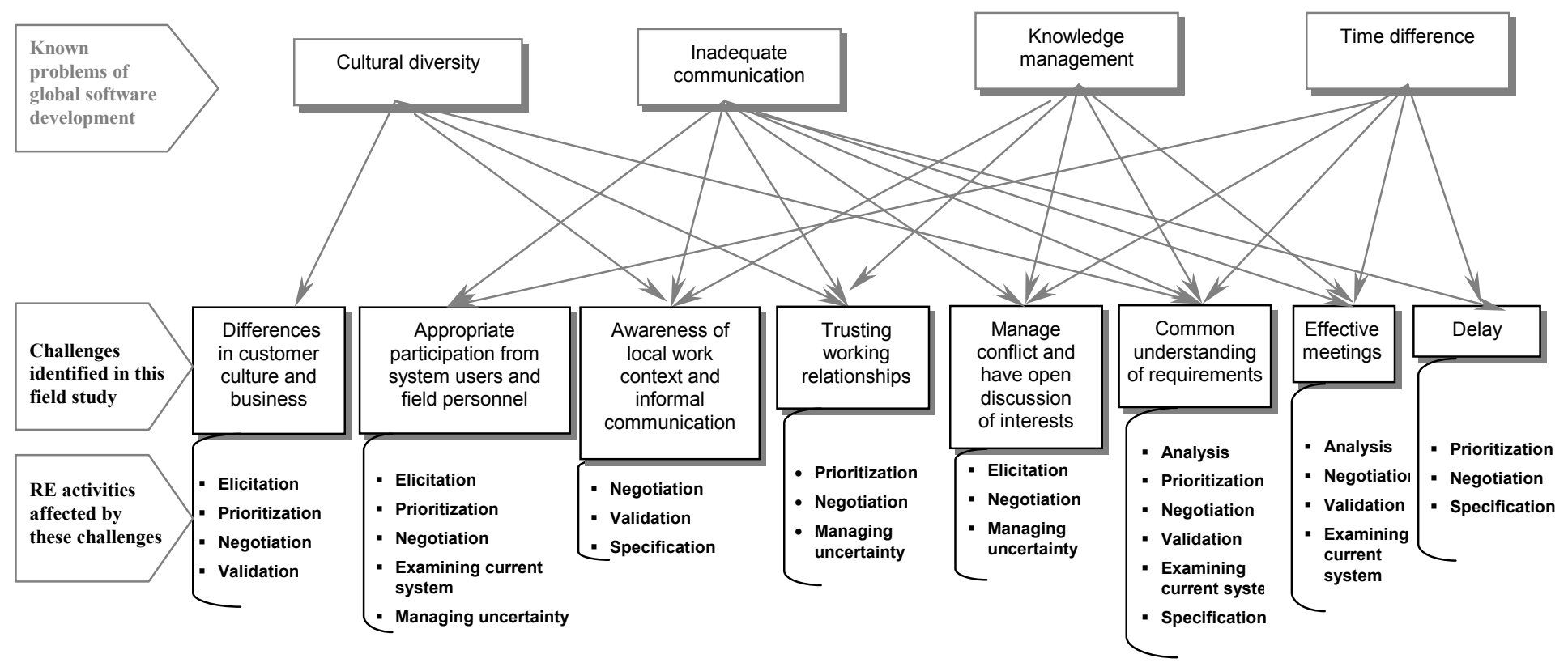

Figure 1. A model of impact of challenges and the affected RE activities due to problems of cultural diversity, inadequate communication, knowledge management and time differences in global software development

- Knowledge management. The sheer quantity of information and knowledge bout requirements from multiple sources at remote customer sites was not appropriately shared with the developers.

- Cultural diversity. Differences in stakeholders' language and national culture affect global collaboration. Equally important in this study was the impact of differences in organizational and functional culture. Not only did remote sites develop their own organizational culture, but also the distance widened the gap between the different functional departments of the organization (marketing, business management, development and engineering). This had a significant impact on achieving a common understanding and negotiation of requirements.

- Time difference. The large distribution of stakeholders introduced large time-zone differences and allowed little overlap available for synchronous collaboration. Hence asynchronous channels were predominant in the communication, complemented by regular teleconferencing calls. Synchronous meetings across continents are always awkward for at least one site either too early or too late in the day, and involve someone having to compromise on their work schedule.

These "generic" problems had created specific difficulties in conducting RE activities and they are described in the second layer of the model. These "challenges" were arranged and labeled such to provide a minimum but meaningful array of distinct and yet possibly overlapping categories. They are described in the next sections, one by one (left to right in Figure 1), together with their impact on RE activities (as outlined in the third layer of the model). The model describes a complex phenomenon, and its arrows show only direct relationships between problems and challenges in the first and second layer respectively.

\subsection{Diversity in customer culture and business}

Although managing requirements from a large customer base is a fundamental problem in RE, customers' geographical distribution exacerbates the problems associated with a large (and conflicting) set of requirements created by the use of the system in diverse market, national, and corporate cultures.

Firstly, customers' language is a critical factor that directly impacts activities such as requirements elicitation and validation since language barriers affect the transfer of knowledge of requirements to system analysts. This challenge was particularly significant at GDS, where nonEnglish speaking customers are located in Asia, Europe and South America.

Additional challenges emerged at several levels: market trends may differ by market segment; differences in national culture often lead to requirements to be meaningful in the context of certain cultural beliefs and values (e.g. some countries may value stability and ask for a requirement only because it was in previous releases, when other clients favor new features in the system for continuous progress).

Furthermore, distance increases the likelihood of diversity in corporate culture and subsequently system operational environment. This was an important issue for SPI in particular, indicating that even when customers speak the same language differences in culture may cause problems to global software enterprises. The different corporate cultures in the five American customer sites created significant conflicts in requirements, the 
customers speaking the same language had clear difficulties in agreeing on how the system will be used in their own organization.

These factors contribute to a fundamental problem in RE [18]: requirements being expressed using diverse terminologies and level of detail, thus making the analysis for consistencies, conflicts, and redundancies difficult. This leads to a significant challenge: the prioritization and negotiation of customer requirements for a particular release, in the context of specific business and strategy requirements, and limited development resources. Pinning down requirements stands as a real challenge because of the difficulty of making trade-offs on a large list of diverse requirements in the face of uncertainty. Although models of market-driven requirements engineering processes that promote a good communication with marketing departments in the prioritization of requirements exist [17], the application of these models at organizations such as GDS is problematic due to challenges in appropriate communication with field support departments, as described next.

\subsection{Achieving appropriate participation of system users}

Not only did the geographical distribution of customers bring diversity in requirements, but also it represented a significant barrier to interactions between system users, analysts and developers, affecting the "problem solvers" active participation in the gathering, analysis, prototyping and validation of requirements. Part of the problem is that the RE process does not require users' active involvement in the specification of requirements. Global organizations such as GDS or SPI facilitate the communication with system users through intermediate groups such as field support personnel at GDS or a Project Office group at SPI, when customers are more localized in one geographical area. Although this strategy has a perceived positive effect in filtering customer requests such that "developers can get on with building the system", it is in fact problematic in achieving an acceptable transfer of requirements knowledge from users to developers. Heavy reliance on indirect communication with system users in eliciting requirements leads to the fundamental problem of misinterpretation of and distorted information about requirements. Because examining the current system in the users' environment is not feasible, indirect sources of requirements lead to a lack of understanding of the rationale behind requirements and ways in which users are using particular functionality.

Distance puts an additional barrier on removing these organizational problems if there was an interest from the customer to be involved. Customer visits to the development sites are rare or non-existent. Due to the large time difference between Australia and US or
Europe, there is a tendency to rely on written documentation, found as very poor to communicate clear requirements. As one field manager put it, "if the engineers were closer to us, I would develop a 'visit your customer' plan". At SPI, a one-week visit of customers at the Australian development site, 2 years in the development, was perceived as very effective and essential in achieving an understanding of requirements not possible through tens of iterations of requirements documents over almost two years! As discussed in Section 4.4, the face-to-face contact during these meetings are critical in establishing an appropriate level of trust between customers and developers.

\subsection{Lack of informal communication and reduced awareness of local working context}

One of the most striking features of distributed groups is the lack of informal communication, i.e. spontaneous and "corridor talk" conversation [11]. Stakeholders have reduced opportunities to know "what is going on at the other site", because of insufficient familiarity with the activities of remote group members and background information that make work contexts meaningful. Observations revealed subtle consequences of the lack of informal communication and lower awareness of local context on the relationship between different stakeholder groups at both organizations.

Informal communications is very important for coordination of work and for learning the culture of an organization. It is also crucial for the perpetuation of the social relations that underlie collaboration and generally, in any situation that communication is required to resolve ambiguity [9]. Requirements Engineering is indeed among such situations where electronic media make it difficult to transmit equivocal messages, whose ambiguity in meaning permits multiple interpretations of requirements.

Through informal communication, the reaction to a requirement-related issue is propagated much quicker locally than across sites. There is significantly greater ability to tap into immediate knowledge in co-located development. In both organizations, requirements-related communication between remote sites is mostly done through "formal" channels, i.e. the bi-weekly meetings, when the communication is focused on urgent issues and leaves little room for small talk. Outside these meetings, the communication between stakeholders is primarily channeled through the non-interactive email, or phone calls, when improper knowledge management techniques make the communication ineffective. Reliance on asynchronous channels contributes to issues identified at one site, small or big -- which may crop up on a daily basis -- go unrecognized at the other site, and thus unresolved for a long time. 
At the same time, informal communication within one site has a positive impact on the local negotiation process. Whenever there is the need to address requirementsrelated issues -- not only it is easy to walk to someone's desk but also there is more minute-by-minute understanding of people's reaction to what one says, so one can easily adjust what is being discussed. Moreover, the knowledge of local working context provides someone with an indication of someone's position, information beneficial in negotiations. Further, the face-to-face interaction on a daily basis has also a natural effect: the creation of coalitions. Cohesion is more difficult for crosscultural teams [1], groups at one site developing a shared view and relating more easily to issues being discussed during formal decision-making. A psychological separation between the development group and the other key players in decision- making (i.e. BM group at GDS and Project Office at SPI) was observed, expressed in feelings of "us versus them".

To make matters worse, diminished awareness of local working context and ineffective knowledge management across sites do not blend well with differences in organizational culture. A direct consequence is the difficulty in defining roles and expectations in the RE process. At GDS, lack of clear guidance on the process (from the mother-organization) led to development group following its own customized RE process. Although the Project Manager had an instrumental role in defining the customized RE process, inadequate communication led to neither the process nor the rigor in developing the requirements was fully understood by the other decision makers in US. For example, when not enough information was communicated following a review of RS, this not only resulted in BM showing insufficient appreciation of development's effort and processes followed to produce the RE artifacts, but also in developers not fully appreciating the pressures and business context in which BM operates in US.

A similar situation was observed at SPI, however magnified by weaknesses in project management. While it is known that global projects bring additional challenges to project management [3], at SPI there was an unanimous dissatisfaction with the Project Manager's skills in identifying a clear direction for the RE process and roles to support it. The concept of "not working together" emerged when the impact of distance was discussed with the stakeholders, and resulted in the attitude of "there is no knowledge of what the others are doing, or should do". For more than a year in the project, the project stakeholders at SPI had not clear understanding of the other's role in the development of requirements. While the development group in Australia kept asking for requirements to be defined by the Project office (in US) through their communication with the customers, the Project office members were waiting for system components to be delivered, based upon "understood" requirements.

In both projects, the lack of well-defined roles and expectations led to misinterpretation of actions, due to stereotyping about cultures and working styles. It often generated negative attitudes, exacerbated by existing conflicts due to political struggles, and hence changed the atmosphere of the requirements negotiations.

\subsection{Reduced level of trust}

"The most trusted people are those that are most accessible or available" (PM at SPI) is evidence that indicates that more frequent interaction leads to better trusting the person. While co-located teams can build trust through formal and informal face-to-face interactions, distance is an impediment to building trust relationships [3]. The role of the "coffee talk" is important again, as one stakeholder simply put it "at distance it is harder to become a team... you would like to talk to [other key stakeholders] more often with a cup of coffee; you need to know each other personally to trust each other, to see the value of a person, to become engaged and committed, to follow the same agenda."

As a result, at GDS cross-site negotiation and prioritization meetings are characterized by extra caution and consciousness in making commitments, which some described as "guarding themselves against things that may be taken to their disadvantage", as well as by a reduced level of trust in arguments, i.e. "was there a hidden agenda?" Managing uncertainty was difficult because often the information was left deliberately ambiguous. For example, the fear for scrutiny led to restricted information about development estimates being communicated by BM, damaging the level of trust across sites.

An additional challenge in building trusting relationships is the historical relationships between remote stakeholder sites. Like a co-located team, a distributed team develops trust [3] slowly as it progresses through the evolutionary stages of working together, stages of forming-storming-norming-performing in Tuckman's team performance model [20]. There is an interesting mapping between these stages and system development, in that the early phases of defining requirements and managing significant uncertainty in the project corresponds to stages where trust relationships may not exist (if prior experience on projects is non-existent), but potentially shaped at later stages. Existing personal relationships play a crucial role in developing a certain level of trust. Data from both global organizations provide evidence in this direction.

At GDS, while trust relationships existed for some team members due to involvement in past projects, it was a clear challenge for some newly assigned team members. The project manager's case presents a clear example of where lack of personal relationships with the remote 
stakeholders had a significant effect on the level of trust attributed to his work and contributions to the RE process. He was new to the organization and hence it was extremely difficult for him to "manage" the diverse demands on the projects and to gain trust in his work and arguments.

Evidence from SPI supports this finding further. None of the stakeholders in the development and project office groups had personally known each other or worked on projects before. Although once-a-month meetings at the Project office site in US would include one or two members of the Australian group, for most Australians the working relationships were established via email or teleconferencing calls. Besides some conflicts at personal level - exacerbated by remote collaboration, interview data suggests a pattern of mistrust that the other group understands the requirements, together with stereotypical attitudes that the Australian development site delivers late and less than optimal quality software.

Finally, establishing face-to-face interaction with the Australian development groups was equally important for customers in both global organizations, in building their trust that the customers' needs are being addressed in a professional manner.

\subsection{Difficulty in managing conflict and having open discussions of interests}

Distance makes it more difficult to deal with problems of organizational, political and social nature. As one engineering manager noted: "it's hard to do strategic thinking at a distance, to bring everyone on the same page... if you get all stakeholders on a three-day conference to do strategic planning, you'd save months of biweekly requirements calls".

While conflicting viewpoints is a fundamental problem in requirements engineering [15][18], distance makes it more difficult to manage conflict. One of the most reported challenges was the ability to deal with the different and most often conflicting viewpoints in the development. Different demands (customers- or businessdriven) are placed on system development and distance diminished the ability to openly discuss the different stakeholders' interests. Conflicts surface in the area of requirements themselves as well as with respect to approaches to the development and management of requirements. The negotiation of trade-offs in an open forum is difficult enough in co-located development, and it is significantly dependent on the quality of the stakeholders' communication, knowledge and project management techniques in distributed structures. The problem is largely due to an inadequate channeling and management of preferences and expectations.

Firstly, conflicts in requirements are difficult to manage at a distance because demands generated from different sources are often channeled through lateral and not direct communication. For example, the communication of requirements as a result of some stakeholders' hidden agenda was not done publicly - but by use of private email messages or telephone calls. The large time-zone difference makes the time window available for phone calls to discuss emergent issues too short; most often these issues do not surface during formal decision-making teleconferencing calls (and when they do, the mute button is too often used), making them stay dormant for long time, contributing to even more conflict. As a consequence, there is a perception that unresolved conflicts have perpetuated along the years, only damaging the relationship between the distributed stakeholders. This has a direct impact on the ability to elicit hidden requirements and to manage inherent uncertainties, ultimately damaging the communication between remote stakeholders.

Second, another conflict of viewpoints difficult to manage in global organizations is caused by the difference on organizational cultures at local sites, and is related to how requirements are being developed in the project. Often development labs such as the two studied in Australia need to comply to processes defined in the global organization. This leads to the local processes may not be understood at remote sites and, as detailed in the next section, and disagreements over processes and outcomes in developing requirements often occur. For example, at GDS attitudes such as "we do not understand how these requirements were developed and what functionality is in fact delivered" were observed. The same aspect was noted at SPI, (unfortunately) to an even greater extent: although the project office and development groups were supposed to work together in system development, a major clash between the two organizational cultures led to significant conflicts in the methodology to requirements engineering. Attitudes such as "we don't agree on the methodology, this is not how we are doing things over here" were observed because the two groups had different ways of project execution; from the perspective of the development group in Australia, the role of the project office group in US was to coordinate the gathering and negotiation of requirements from the customers. On the other hand, however, the project office had advocated a minimal role in developing requirements, although there was recognition of their expertise in the customers' problem domain. This conflict has resulted at SPI in no group taking ownership of requirements.

Although these types of conflicts may exist in colocated development, it appears that their resolution is significantly more difficult in the absence of a common working environment. Group members are depleted by the ability to share a common view towards system development methodologies, or become accustomed and accept a particular RE process. 


\subsection{Difficulty in achieving common understanding of requirements}

The same way conflicts in RE processes and methods were rooted in differences in organizational culture, achieving a common understanding of requirements was caused mainly by differences in stakeholders' functional role in system development as well as ineffective remote communication in bridging this gap.

In both organizations, the development groups had to rely on the communication with a "surrogate customer", which proved to be a poor substitute when it came to providing detailed requirements or information about the system's operational environment. Projects both at GDS and SPI suffered from the classical problem of the communication gap between customers and developers [8]. Different functional groups were involved in defining the requirements for the actual system end-users: the business management and development groups at GDS and the project office and development groups at SPI. This led, particularly, to the requirements communicated to the developers not providing sufficient detail for decision-making, prioritization, and negotiation, let alone design activities.

In both cases, the Australian development groups took leadership in developing a requirements specification to capture their understanding of the functionality. The success of this specification in aiding the requirements prioritization and negotiation is yet to be proven. While there is some reluctance in this direction at GDS, stakeholders at SPI expressed a general dissatisfaction with the usefulness of the requirements specification in documenting any shared view of requirements. The developers' attempt to communicate with the customers indirectly - through the project office- resulted in lengthy reviews and iterations of specification documents, with no confidence that progress towards a shared understanding is being made.

\subsection{Ineffective decision-making meetings}

Although there are discussions between stakeholders outside formal and shared forums, decisions are agreed upon in formal meetings. These meetings are perceived equally important and challenging.

Firstly, there is a fair degree of pressure in setting up these meetings. Due to the geographical distance, they inevitably require considerable a priori preparation. They require the involvement of key decision makers, i.e. Business Management members in US and Development Managers in Australia (at GDS) and Project office and the Development group (at SPI respectively). While at SPI face-to-face meetings in the US-based Project Office once a month involve one or two Australian representatives are complemented by weekly teleconferencing project reviews, at GDS all weekly meetings occur via teleconferencing.

Secondly, communication and knowledge management problems impinge on the effectiveness of these meetings and most of the challenges discussed thus far in the paper are directly related to the quality of these meetings. When asked which of the challenges of distance to address first in our research, the response was almost unanimous at GDS: "improve requirements meetings!"

Part of the problem is the communication medium used is these meetings. When teleconferencing is the main medium that bridges several separate locations, there is also the need to send supporting documents to all stakeholders well prior to the meeting, and express yourself concisely and clearly: not only these people have busy schedules, but also the small time overlap between the continents limits the time available to address issues gathered before (i.e. during at least couple of weeks). Further, the lack of visual contact contributes to a lowered awareness of presence and group behavior at the remote sites. Participants can join the group at later times and, without a good facilitator, their presence might not be announced. This leads to problems in knowing who can be addressed with regards to a particular issue and thus effective participation is diminished. The mute button is also naturally used, and adds to the creation of coalitions and damaging the already low possibility of having open negotiations. Further, there is no means of synchronously creating and sharing work artifacts. Access to a whiteboard for sketching ideas is limited to one site with no ability for collaboration. Hard-copy documents such as the RS are used as vehicles for discussion and decisions. However, distance makes it more difficult to detect differences in shared hard-copy documents, i.e. page numbering. This often led to lowered level of participation in discussions/decisions about requirements (due to attention being paid in locating such requirements!).

Another interesting aspect observed in these meetings was the need to access supporting documents that contained information relevant to requirements, such as email messages or documents received from the customers. Questions such as "what do we know about this requirement?" or "Have we discussed it before?" managing the information from customers and market; the lack of a repository storing the history of issues regarding a particular requirement resulted in issues and decisions being revisited and delayed for weeks.

Although the communication and knowledge management aspects of these meetings are critical and exacerbated by distance, there are other aspects that contribute to meetings' effectiveness. They are of human, social, organizational and management nature: factors such as timely exchange of documents to allow reading, adequate stakeholder preparation for the meetings, dominance by some group members, and ultimately 
following an agenda are as important as those discussed above. As one manager put it, "if you don't have an agenda it is often too easy to attend to the most recent customer complaint or requirement, rather than having a strategic discussion of "where is the product going?"”

\subsection{Delay}

Speed is regarded as one of the most important success factors in modern high technology businesses and it is becoming of concern in global software development [9]. In both organizations, whenever requirements-related issues arose that required cross-site communication, options included sending an email, making a phone call or waiting for a formal requirements meeting to take place. Although delay was not reported as an impediment to communication across the US sites, it was a major concern for the development site in Australia. One explanation is the difference in time zones. BM members reported that they spend 2-3 hours in a row in discussing requirements. Synchronous communication is good in resolving misunderstandings, and small issues before they become bigger problems. Due to a larger time difference, however, this is limited in the communication across continents; development engineers need to rely mostly on asynchronous communication, which leads to situations where "a small issue with a requirement can take days back-and-forth discussions over email to resolve, if not complicated."

A significant impact was observed on the requirements negotiation and prioritization activity. The ineffectiveness of formal decision making meetings combined with the use of email in resolving issues led to decisions being delayed and issues remaining unresolved longer than necessary. At the time of this research report, the list of features being considered in the current release is still being negotiated, six months beyond the proposed deadline.

\section{Conflict, culture and distance}

We now revisit some of the important findings of the case study specifically related to culture and conflict and will discuss the interplay between various forms of culture and conflict as well as the impact on the requirements engineering process and products within global software teams.

We observed conflicts in agreeing requirements as well as in deciding on approaches to the RE process that should be followed within the organizations. These types of conflicts can be attributed to ineffective channeling of information and management of requirements process as well as the existence of different cultures (national, functional and organizational) at the remote sites. This in turn is impacted by distance that prevents a thorough understanding of different cultures to be achieved by distributed stakeholders.
One of the important challenges faced was dealing with conflicting requirements from multi national customer groups with differences in cultural beliefs and values. For example for some cultures stability is very important. Hence when it comes to requesting requirements for a new release, they may ask for requirements purely because they were in the previous release of software. Customers from another cultural background, however, may ask for entirely new features just because they want to be up to date and progressive in their approach to technology. These competing and conflicting cultural values impacts on the ranking, prioritizing and negotiating of requirements.

In this study we observed conflicts in common understanding of requirements which can be attributed to differences in both functional and national cultures. Ee observed that differences in functional cultures (manifested itself in the lack of well-defined roles and expectations of different functional teams), led to misinterpretation of actions. For example, in SPI, development team in Australia expected the project office team in USA to have continued involvement in the RE process and to play an active role in clarification of requirements by liaising with the customer. The Project office in USA, however, thought that their job is complete after the contract has been signed and it was up to the development to adopt a process and clarify the requirements. Hence, they did not provide any directions for the process even though the development team in Australia expected it. This created an enormous degree of conflict between two teams.

Attitude towards hierarchy in different national cultures ("power distance", in Hofstede's terminology [12]) is an important factor to be considered in studies of this kind. This factor has to do with how people perceive equality and relationships with superiors and subordinates [3]. Different attitudes towards hierarchy can contribute to emergence and resolving of conflict. This is important in RE particularly when stakeholders are committing to requirements or occasions where they have to decide on which features to be included in the system to be built. In both organizations studied, the requirements decisions generally came from management staff in USA since they are also acting as surrogate customers. Australian stakeholders prefer to be more involved in the decision making. This finding is in agreement with Hofstede's [12] results on classifying Australia as a relatively small power distance country where employees express a preference for a consultative style of decision making. For example in GDS, the development team often said that they needed to know why a feature was suggested by the program management, while the management in USA saw their role as only the determiner of requirements and did not see any need to involve development team in initial decision making. Research into managerial values [19] based on 
data comparing 12 different countries including Australia and USA, shed an interesting light on this issue. Spillane [19] identified and measured five values in these countries and found that in four of these values Australia and Americans hold very similar views. However, the fifth value - whether society should be structured according to achievement or other criteria - was where the two groups of managers diverged. Americans are found to be the most achievement-orientated society and their managers are strong believers in performance, and rewarding people who perform and penalizing those who don't. In our findings we observed frustration of the US management in late delivery of software releases by the Australian developers. We wondered if this may be the reason why Americans do not involve the Australian developers more in the decision making.

Any type of conflict, if left unresolved for a long time affect the trust between various stakeholders. Unresolved conflicts and ambiguities in requirements in both organizations in some cases resulted in more conflicts and less trust. This is a clear indication of differences between organizational cultures at different sites that manifested itself in different expectations and conflict in the role of people in producing RE artefacts as well as in their involvement in various RE activities. It was unclear who should be doing what in the RE process. For example lack of a thorough understanding of corporate culture (that in this case includes a specific RE process) at a higher level caused GDS to adopt their own RE process. But because the details of the new RE process at GDS was not communicated well to the management in USA the effort made by GDS staff in analyzing and elaborating requirements was not fully appreciated by the USA counterparts. At SPI, even two years in the project, the roles of various stakeholders in the elicitation, analysis and specification of requirements were not understood or even defined, let alone agreed upon.

In our study the concept of trust has emerged as intrinsically related to cultural values. We observed that trust was more important to the Australian teams than to the American teams. This may be because Australia can be considered as what culture social scientists refer to as high context culture. High-context cultures communicate a message in which most of the information is in the person, the surroundings, and the social perspective. Much of what is communicated is not said [3]. For these cultures the relationship of trust is very important and lack of trust may contribute to emergence of conflict as well as getting in the way of resolving conflict.

Face-to-face communications play a very important role in increasing the understanding of participants' culture, be it functional, organizational or national culture, and hence potentially increases the level of trust. Our findings in this study confirm that face-to-face meeting significantly contributes to appreciating the value of each person in the team, becoming engaged in and committed to achieving the business goals, being able to follow a common agenda, becoming familiar with individual personalities, enabling people to show respect and increasing trust amongst team members. More frequent informal interactions thus lead to improving trust among team members. Distance was found to be an impediment to facilitating sufficient interactions and hence does not help in building a trusting relationship in multi-site development.

Furthermore, history of personal relationship plays a significant role in developing a certain level of trust. Although a few of the GDS staff in Australia have been involved in previous projects with their colleagues in USA or in the case of SPI, monthly meetings in US included one or two members of the Australian group, for most Australians the working relationships were established via email or teleconferencing calls. Besides some personality conflicts exacerbated by remote collaboration, interview data also suggests a pattern of mistrust with respect to understanding of requirements.

Findings in some cases indicated stereotypical attitudes between Australians and their American counterparts. This prompted us to carefully examine the issues attributed to national cultures as described by Hofstede [12]. Hofstede's seminal work used survey data collected over 11 years from IBM personnel in 40 countries (including Australia and USA), on the topic of work values. He isolated attitudes of people towards their work along five "dimensions of culture". We compared Hofstede's data collected for Australia and America within these dimensions and found that both countries rank very closely in almost all scales produced. In other words, it may be inferred that in fact national cultures of USA and Australia are close as far as these dimensions represent. We feel that perhaps national culture is being used as a scapegoat in both organsiations to cover up ineffective management practices at various levels. Hofstede states that confusing the level of the individual with the level of the society (known in the social sciences as ecological fallacy) amounts to a confusion between personality and culture. This is a difficult topic to study especially in IT because unlike social sciences literature there is hardly any conclusive evidence. We do not wish to make a strong claim or generalisation in any way. What is important, however, is the need to achieve a better understanding of the interplay between culture (national, functional or organizational) and conflict in distributed teams and how distance impacts on its resolution.

\section{Conclusion}

In this paper we reported the findings from a case study that identified important challenges that multi-site development teams face in eliciting, analyzing and 
specifying requirements across geographical, cultural and time zone boundaries. A common theme that emerged across the two organizations studied was the interplay between culture and conflict and the impact of distance on the ability to reconcile different viewpoints with regards to requirements as well as requirements processes.

While inadequate communication significantly impacts the bridging of geographical distance between stakeholders, the cultural differences cannot be considered less significant. The resolution of conflicts as found in this case study not only requires good communication in the project but also an understanding of these cultural differences and how they can be overcome. We believe that players in global virtual collaboration need to be aware of cultural differences and invest considerable effort in training about different cultures to improve their approaches to conflict resolution during requirements management. There is also a need for researchers to pay due attention to developing RE processes that address these crucial issues [23].

\section{References}

[1] Adler, N.J. International dimensions of organizational behavior. Southwestern College Publishing, 1997

[2] Al-Rawas, A. and Easterbrook, S. Communication problems in requirements engineering: a field study, Proc. of Conf. on Prof. on Awareness in Software Engineering, London, 4760, 1996

[3] Carrmel, E. Global software teams, Prentice Hall, 1999

[4] Checkland, P. Systems Thinking, Systems Practice, Wiley, 1981

[5] Damian, D. Empirical studies of computer support for requirements negotiations, $P h D$ thesis, 2001

[6] Damian, D.E, Eberlein, A., Shaw, M.L.G. and Gaines, B.R. Using different communication media in requirements negotiation, IEEE Software, May/June, 28-36, 2000

[7] Damian, D. and Zowghi, D. The impact of stakeholders' geographical distribution on managing requirements in a multi-site organization, in Proceedings of the Requirements Engineering (RE02), Essen, Germany, 2002

[8] El-Emam, K. and Madhavji, N. A field study of requirements engineering practices in information systems development, $2^{\text {nd }}$ Int'l Symposium. Requirements Engineering, 1995

[9] Fish, RS., Kraut, RE., Root, RW., Rice, RE., Evaluating Video as a technology for informal communication, in ACM proceedings on Human Factors in Computing Systems, June 1992.

[10] Herbsleb, J., Mockus, A., Finholt, T.A. and Grinter, R.E. An empirical study of global software development: distance and speed, Int'l Conf. on Software Engineering, Toronto, 2001

[11] Herbsleb, J. and Moitra, D. Global software development, IEEE Software, March/April, 16-20, 2001
[12] Hofstede, G. Cultures and Organizationa, software of the mind, Intercultural cooperation and its importance for survival, Harper Collins publishers, 1994.

[13] Holtzblatt, K. and Beyer, H.R. Requirements gathering: the human factor, Comm. of the ACM 38(5), 31-32, 1995

[14] Macaulay, L. Requirements Engineering, Springer, 1996

[15] Nuseibeh, B. and Easterbrook, S. Requirements engineering: a roadmap, Int'l Conf. on Software Engineering, Ireland, 2000

[16] Ramesh, V. and Dennis, A.R. The object-oriented team: lessons for virtual teams from global software development, Proc. of HICSS35, Hawaii, 2002

[17] Regnell, B., Host, M., Och Dag, J.N., Beremark, P. and Hjelm, T. An industrial case study of distributed prioritization in market-driven requirements engineering for packaged software, Requirements Engineering Journal, $6(1), 51-62$

[18] Sommerville, I. and Sawyer, P. Requirements Engineering: A Good Practice, Wiley, 1997

[19] Spillane, R. (2002). Getting a life: Aussie management style, Macquarie University News, Australia, April 2002

[20] Strauss, A. and Corbin, J. Basics of Qualitative Research: Grounded theory procedures and techniques, Sage, 1990

[21] Tuckman, B. Developmental sequence in small groups, Psychological Bulleting, 63, 384-389, 1965

[22] Yin, R. K. Case Study Research, Sage, 1994

[23] Zowghi D. "Does Global Software Development Need a Different Requirements Engineering Process?", Proceeding of International Workshop on Global Software Development, (In conjunction with ICSE 2002, Orlando, Florida). 